\title{
Genomic variations in paired normal controls for lung adenocarcinomas
}

\author{
Li-Wei Qu ${ }^{1, *}$, Bo Zhou ${ }^{1,2, *}$, Gui-Zhen Wang ${ }^{1}$, Ying Chen ${ }^{1}$ and Guang-Biao Zhou ${ }^{1}$ \\ ${ }^{1}$ Division of Molecular Carcinogenesis and Targeted Therapy for Cancer, State Key Laboratory of Membrane Biology, Institute \\ of Zoology, Chinese Academy of Sciences, Beijing 100101, China \\ ${ }^{2}$ University of the Chinese Academy of Sciences, Beijing 100049, China \\ *These authors have contributed equally to this work \\ Correspondence to: Guang-Biao Zhou, email: gbzhou@ioz.ac.cn \\ Keywords: lung cancer; genomic variations; counterpart normal control; tobacco smoke \\ Received: July 29, $2017 \quad$ Accepted: September 23, $2017 \quad$ Published: October 24, 2017 \\ Copyright: Qu et al. This is an open-access article distributed under the terms of the Creative Commons Attribution License 3.0 \\ (CC BY 3.0), which permits unrestricted use, distribution, and reproduction in any medium, provided the original author and source \\ are credited.
}

\section{ABSTRACT}

Somatic genomic mutations in lung adenocarcinomas (LUADs) have been extensively dissected, but whether the counterpart normal lung tissues that are exposed to ambient air or tobacco smoke as the tumor tissues do, harbor genomic variations, remains unclear. Here, the genome of normal lung tissues and paired tumors of 11 patients with LUAD were sequenced, the genome sequences of counterpart normal controls (CNCs) and tumor tissues of 513 patients were downloaded from TCGA database and analyzed. In the initial screening, genomic alterations were identified in the "normal" lung tissues and verified by Sanger capillary sequencing. In CNCs of TCGA datasets, a mean of 0.2721 exonic variations/Mb and 5.2885 altered genes per sample were uncovered. The $\mathrm{C}: \mathrm{G} \rightarrow \mathrm{T}: \mathrm{A}$ transitions, a signature of tobacco carcinogen $\mathrm{N}$-methyl- $\mathrm{N}$-nitro- $\mathrm{N}$-nitrosoguanidine, were the predominant nucleotide changes in CNCs. 16 genes had a variant rate of more than $2 \%$, and CNC variations in MUC5B, ZXDB, PLIN4, CCDC144NL, CNTNAP3B, and CCDC180 were associated with poor prognosis whereas alterations in CHD3 and KRTAP5-5 were associated with favorable clinical outcome of the patients. This study identified the genomic alterations in CNC samples of LUADs, and further highlighted the DNA damage effect of tobacco on lung epithelial cells.

\section{INTRODUCTION}

Comprehensive sequencing efforts over the past decade have confirmed that cancer is a disease of the genome [1]. Genomic alterations in cancers include point mutations, insertions and deletions (indels), structural variations, copy number alterations, epigenetic changes, and microbial infections. Abnormalities in oncogenes and tumor suppressors act as driver mutations to initiate the onset and progression of cancers. Technically, these alterations are identified by comparing the cancer genome sequence with a reference human genome and excluding those also found in normal controls (counterpart normal tissues or peripheral blood) and single nucleotide polymorphisms (SNPs) [2]. This strategy is effective to uncover somatic genomic alterations in tumor tissues. However, whether there is any genomic alteration in counterpart normal controls (CNCs) but not cancer tissues, remains unclear.

Lung cancer is the most common cause of cancer related mortality worldwide [3] and can be divided into small cell lung cancer (SCLC) and non-small cell lung cancer (NSCLC). NSCLC comprises of lung adenocarcinoma (LUAD), lung squamous cell carcinoma (LUSC), and large cell carcinoma [4]. It is estimated that $90 \%$ of lung cancer deaths are caused by cigarette smoke [5], whereas SCLC and LUSC are most strongly linked with smoking [4]. Since the late 1980s, LUAD has 
become the most common type (40\%) of lung cancer that is related to cigarette smoke due to the changes in cigarette composition and filtering which favor adenocarcinoma histology [6]. There is also a proportion of LUADs that is not associated with smoking [4], while air pollution represents another cause of lung cancer which induces somatic mutations in the genomes [7]. The cellular injury produced by smoking involves the whole respiratory tract $[8,9]$; in the initial phase of lung carcinogenesis, injury is repaired by stem/progenitor cells, forming a clonal group of self-renewing daughter cells. Accumulation of genetic alterations in the premalignant field results in proliferation of the cells and expansion of the field, gradually displacing the normal epithelium and developing into a malignant neoplasm [10-12]. Genomic alterations of LUADs had been characterized and druggable targets had been unveiled [13, 14]. However, very few studies systematically dissect genomic mutations specifically occurred in "normal" lung tissues and their roles in carcinogenesis, and no study reports how the adjacent tissues escape from developing into malignant neoplasms.

In our work analyzing somatic genomic mutations in air pollution-related lung cancers [7], we noted that when compared with the reference human genome, some variations were seen in counterpart normal lung tissues but not cancer samples (Table 1). To expand these observations, we further analyzed the Cancer Genome Atlas (TCGA) lung cancer genome data of 513 CNCs and counterpart tumor samples (Supplementary Figure 1). Our results showed that the $\mathrm{CNCs}$ did harbor some genomic variations.

\section{RESULTS}

\section{Identification of $\mathrm{CNC}$ somatic genomic variations}

The genomic DNAs of paired normal lung tissues and cancer tissues were sequenced to an average of $42.89 \times$ (range, $30.07 \times-78.70 \times$ ) coverage and 66.04× (range, $61.02 \times-74.64 \times)$ coverage, respectively. Twenty nucleotide substitutions and 7 small insertions and deletions (indels) were found in the 11 LUADs genomes (Table 1). Variations in 6 representative genes in the normal lung tissues were validated by RT-PCR assays and subsequent sequencing (see Supplementary Table 1 for sequence of primers), and the results confirmed the existence of CNC alterations (Figure 1). For example, the nucleotide at chr18: 22806878 of ZNF521 of hg19 is C; but two peaks $(\mathrm{C}$ and $\mathrm{T}$ ) of approximately equal peak height were seen in sequence of normal lung tissues, whereas a high peak of $\mathrm{C}$ and a low peak of $\mathrm{T}$ were detected in tumor samples of the patient (Figure 1A, left panel). This change might lead to P335L substitution in the encoded protein. Sequencing results using another set of primers confirmed the existence of $\mathrm{T}$ in normal lung rather than counterpart tumor sample of the patient (Figure 1A, right panel). Nucleotide $\mathrm{T}$ at this position of ZNF521 was not found in dbSNP138 common germline variants. The nucleotide at chr17: 76121923 of TMC6 of hg19 is C; but two peaks ( $\mathrm{C}$ and $\mathrm{T}$ ) of approximately equal peak height were seen in sequence of normal lung tissues, whereas a high peak of $\mathrm{C}$ and a low peak of $\mathrm{T}$ were detected in tumor samples of the patient (Figure 1B, left panel). This change might lead to T105M substitution in the encoded protein. Sequencing results using another set of primers confirmed the existence of $\mathrm{T}$ in normal lung rather than counterpart tumor sample of the patient (Figure 1B, right panel). Similarly, the normal lung tissues had a single nucleotide change in CEP250 (Figure 1C), C9orf66 (Figure 1D), and HIST1H1D (Figure 1E), compared to those in the tumor samples, hg19, and dbSNP138. Substitutions of GT by AC were seen in UNC93A in normal lung tissue of a patient with untreated LUAD (Figure 1F).

\section{Analyses of TCGA datasets}

To expand the above observations, the genome sequence of cancer-control paired samples from 513 patients were downloaded from TCGA datasets and carefully analyzed. Among these patients (Supplementary Table 2), 239 (46.6\%) were males and 274 (53.4\%) were females, and the median age was 66 years old (range, $33-$ 88 years). Smoking history was available in 499 patients, among them $425(85.2 \%)$ were current or reformed smokers (persons who were not smoking at the time of interview but had smoked at least 100 cigarettes in their life) and $74(14.8 \%)$ were nonsmokers (not smoking at the time of interview and had smoked less than 100 cigarettes in their life). Adjacent normal lung tissues and peripheral blood were used as normal controls for 135 (26.3\%) and $378(73.7 \%)$ of the 513 lung adenocarcinomas, respectively.

Genomic variations were found throughout the counterpart control genomes. A mean of 0.2721 exonic variations/megabase $(\mathrm{Mb})$ and 5.2885 variants/sample was recorded in the CNCs (Table 2). The normal lung tissues had approximately equal mutations to the blood cells (Supplementary Table 3), while males and females had equal CNC mutations (Supplementary Table 4). We compared the CNC mutations in nonsmokers, current smokers and reformed smokers, and found that smokers harbored more variations than nonsmokers, as reflected by variations/ $\mathrm{Mb}$ (Supplementary Figure 2A), altered genes/sample (Supplementary Figure 2B), synonymous/nonsynonimous variations (Supplementary Figure 2C), and indels/sample (Supplementary Figure 2D). We also analyzed genomic mutations in tumor samples by comparing the cancer genome sequencing with reference and excluding those found in CNCs with the same calling criteria, and reported that tumor samples had much more mutations than CNCs (Table 2 and Supplementary Figure 2E-2H). 
Table 1: Genomic variations in CNCs of 11 patients with LUAD

\begin{tabular}{|c|c|c|c|c|c|c|c|c|c|c|}
\hline Pt \# & Gene & Chr & Start & End & Ref & Alt & Function & Transcript & cDNA position & $\begin{array}{l}\text { Amino } \\
\text { acid }\end{array}$ \\
\hline \multirow[t]{6}{*}{794} & $A T G 16 L 2$ & 11 & 72533157 & 72533157 & A & $\mathrm{T}$ & NS & NM_033388 & c.A461T & p.E154V \\
\hline & CEP250 & 20 & 34092235 & 34092235 & G & A & NS & NM_007186 & c.G6038A & p.R2013Q \\
\hline & MYO1C & 17 & 1371330 & 1371330 & $\mathrm{C}$ & A & NS & NM_033375 & c.G2743T & p.V915L \\
\hline & PNPLA2 & 11 & 824813 & 824813 & G & $\mathrm{T}$ & NS & NM_020376 & c.G1466T & p.S489I \\
\hline & TMC6 & 17 & 76121923 & 76121923 & $\mathrm{C}$ & $\mathrm{T}$ & NS & NM_007267 & c.C314T & p.T105M \\
\hline & ZNF521 & 18 & 22806878 & 22806878 & $\mathrm{C}$ & $\mathrm{T}$ & NS & NM_015461 & c.C1004T & p.P335L \\
\hline \multirow[t]{7}{*}{824} & $A N K S 1 A$ & 6 & 34857302 & 34857302 & - & GGCGG & Cnonframeshift & NM_015245 & $\begin{array}{c}\text { c. } 123 \_124 \text { ins } \\
\text { GGCGGC }\end{array}$ & $\begin{array}{c}\text { p.G41 } \\
\text { delinsGGG }\end{array}$ \\
\hline & $B B S 12$ & 4 & 123664726 & 123664728 & $\mathrm{AAG}$ & - & nonframeshift! & NM_001178007 & $\begin{array}{l}\text { c. } 1679 \\
1681 \mathrm{del}\end{array}$ & $\begin{array}{l}\text { p.560 } \\
561 \mathrm{del}\end{array}$ \\
\hline & C9orf66 & 9 & 214943 & 214943 & $\mathrm{C}$ & $\mathrm{T}$ & NS & NM_152569 & c.C454T & p.R152W \\
\hline & FAM186A & 12 & 50745703 & 50745703 & $\mathrm{~T}$ & G & NS & NM_001145475 & c.A4912C & p.T1638P \\
\hline & HISTIHID & 6 & 26235127 & 26235127 & $\mathrm{C}$ & $\mathrm{A}$ & NS & NM_005320 & c.C35A & p.P12H \\
\hline & $H L A-D R B 1$ & 6 & 32551957 & 32551957 & G & $\mathrm{T}$ & NS & NM_002124 & c.C299A & p.A100E \\
\hline & UNC93A & 6 & 167728776 & 167728776 & GT & $\mathrm{AC}$ & NS & NM_018974 & c.T1210C & p.F404L \\
\hline 760 & $D M K N$ & 19 & 36002386 & 36002386 & $\mathrm{C}$ & $\mathrm{T}$ & NS & NM_001126057 & c.G845A & p.S $282 \mathrm{~N}$ \\
\hline 772 & ZNF259 & 11 & 116658654 & 116658654 & G & A & NS & NM_003904 & c.C53T & p.P18L \\
\hline \multirow[t]{3}{*}{783} & SRAl & 5 & 139931629 & 139931629 & - & $\mathrm{G}$ & frameshift $\mathrm{I}$ & NM_001035235 & 5c.327_328insC & p.V110fs \\
\hline & $T P S A B 1$ & 16 & 1291454 & 1291454 & G & $\mathrm{A}$ & NS & NM_003294 & c.G253A & p.A85T \\
\hline & TPSD1 & 16 & 1306971 & 1306971 & A & $\mathrm{G}$ & NS & NM_012217 & c.A428G & p.H143R \\
\hline 792 & $G P C 3$ & $\mathrm{X}$ & 133087197 & 133087197 & A & $\mathrm{T}$ & NS & NM_001164617 & c.T217A & p.C73S \\
\hline 710 & TPTE2 & 13 & 20048214 & 20048214 & $\mathrm{~T}$ & G & NS & NM_130785 & c.A121C & p.I41L \\
\hline 799 & $C D 33$ & 19 & 51729578 & 51729596 & $\begin{array}{c}\text { CCCAACAACT } \\
\text { GGTATCTTT }\end{array}$ & - & frameshift & NM_001772 & c.711_729del & p.N237fs \\
\hline \multirow[t]{2}{*}{748} & PCMTD1 & 8 & 52733228 & 52733228 & $\mathrm{G}$ & A & NS & NM_052937 & c.C757T & p.R253C \\
\hline & RNASE 8 & 14 & 21526082 & 21526084 & CTG & - & nonframeshift & NM_138331 & c. $31 \_33 \mathrm{del}$ & p.11_11del \\
\hline \multirow[t]{2}{*}{828} & HGC6.3 & 6 & 168376951 & 168376951 & $\mathrm{~T}$ & $\mathrm{C}$ & NS & NM_001129895 & c.A382G & p.M128V \\
\hline & SYNJ1 & 21 & 34003931 & 34003931 & $\mathrm{C}$ & $\mathrm{T}$ & NS & NM_003895 & c.G4213A & p.V1405I \\
\hline \multirow[t]{2}{*}{844} & $M A D C A M 1$ & 19 & 501762 & 501762 & A & $\mathrm{C}$ & NS & NM_130760 & c.A761C & p.Q254P \\
\hline & $N E F H$ & 22 & 29885581 & 29885604 & $\begin{array}{c}\text { AGGCCAAGTCCCC } \\
\text { AGAGAAGGAAG }\end{array}$ & - & nonframeshift & NM_021076 & c. $1952 \_1975 \mathrm{del}$ & $\begin{array}{l}\text { p.651- } \\
659 \mathrm{del}\end{array}$ \\
\hline
\end{tabular}

Alt, alterations; Chr, chromosome; NS, nonsynonymous; Ref, reference human genome.

\section{Nucleotide substitutions in TCGA CNC samples}

Tobacco smoke contains more than 20 lung carcinogens, e.g., nicotine-derived nitrosaminoketone (NNK) [5] and polycyclic aromatic hydrocarbons (PAHs) which are associated with the $\mathrm{C}: \mathrm{G} \rightarrow \mathrm{A}: \mathrm{T}$ transversions in the genomes $[7,15,16]$. We analyzed the nucleotide substitutions in the genome, and found that the $\mathrm{C}: \mathrm{G} \rightarrow \mathrm{T}: \mathrm{A}$ and the $\mathrm{A}: \mathrm{T} \rightarrow \mathrm{G}: \mathrm{C}$ transitions were the most and the second most prevalent nucleotide substitutions in the
CNCs (Figure 2A). In tumor samples, while the $\mathrm{C}: \mathrm{G} \rightarrow \mathrm{A}: \mathrm{T}$ transversions were the most prevalent nucleotide substitutions, the $\mathrm{C}: \mathrm{G} \rightarrow \mathrm{T}: \mathrm{A}$ transitions represented the second most prevalent nucleotide substitutions (Figure 2A). In CNC samples, the $\mathrm{C}: \mathrm{G} \rightarrow \mathrm{T}: \mathrm{A}$ transitions were the most prevalent nucleotide substitutions in nonsmokers, current smokers and reformed smokers (Figure 2B). In consistence with previous report [16], the $\mathrm{C}: \mathrm{G} \rightarrow \mathrm{T}:$ A transitions were the most prevalent nucleotide substitutions in tumor samples of nonsmokers (Figure 


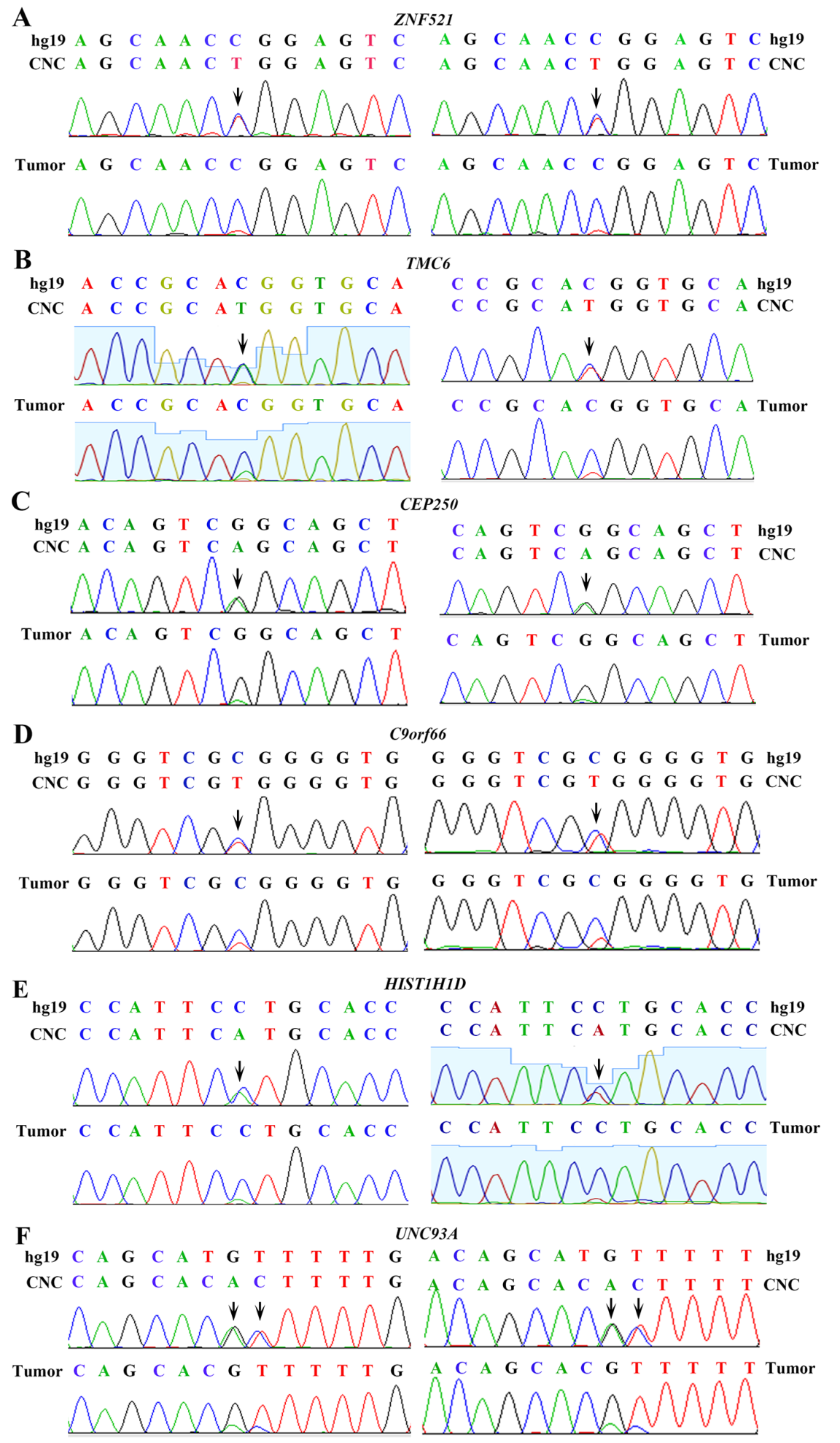

Figure 1: Validation of genomic variations in "normal" lung tissues. Polymerase chain reaction (PCR) and Sanger capillary sequencing were performed using genomic DNAs of the patients and primers listed in Supplementary Table 1. Two sets of primers were used. (A) ZNF521 in "normal" lung and tumor samples of the patient. (B) TMC6 in "normal" lung and tumor samples of the patient. (C) CEP250 in "normal" lung and tumor samples of the patient. (D) C9orf66 in "normal" lung and tumor samples of the patient. (E) HIST1H1D in "normal" lung and tumor samples of the patient. (F) UNC93A in "normal" lung and tumor samples of the patient. 
Table 2: Genomic variations in CNCs and tumor samples of TCGA LUADs

\begin{tabular}{lcccccc}
\hline & $\begin{array}{c}\text { Exonic mutations/ } \\
\text { MB }\end{array}$ & $\begin{array}{c}\text { Variant genes/ } \\
\text { sample }\end{array}$ & $\begin{array}{c}\text { Nonsynonymous } \\
\text { alterations/sample }\end{array}$ & $\begin{array}{c}\text { Synonymous } \\
\text { mutations/sample }\end{array}$ & \multicolumn{2}{c}{ Rearrangements/sample } \\
\cline { 5 - 7 } & 0.2721 & 5.2885 & 4.3723 & 2.8402 & 0.2378 & 0.8129 \\
\hline $\mathrm{CNC}$ & 6.0145 & 141.0132 & 135.7290 & 43.6491 & 6.6862 & 1.9357 \\
Tumor & $<0.0001$ & $<0.0001$ & $<0.0001$ & $<0.0001$ & $<0.0001$ & $<0.0001$ \\
\hline P value & & & & & &
\end{tabular}

$2 \mathrm{C}$ ), whereas the $\mathrm{C}: \mathrm{G} \rightarrow \mathrm{A}: \mathrm{T}$ transversions were the most prevalent nucleotide substitutions in tumor samples of smokers (Figure 2C).

\section{Recurrently altered genes in CNC samples}

We reported that there were 16 genes which had a variation rate of more than $2 \%$ in the $513 \mathrm{CNCs}$ (Figure 2D and Supplementary Table 5). MUC4 represented the most frequently altered gene, which was mutated in 46/513 (8.97\%) of the CNC samples (Figure 2D). FAM90A1, ARSD, MADCAM1, ZNF814, CDC27, and HDGFRP2 were mutated in $35(6.82 \%), 32(6.24 \%), 26$ (5.07\%), $22(4.29 \%), 17(3.31 \%)$, and $16(3.12 \%)$ of the 513 CNC samples, respectively (Figure 2D). TP53, KRAS and $E G F R$ which were frequently mutated in LUADs [13], were mutated in only $2(0.39 \%), 0$, and $0 \mathrm{CNCs}$, respectively (Supplementary Table 5).

MUC4 encodes an integral membrane glycoprotein found on the cell surface and plays a role in tumor
A

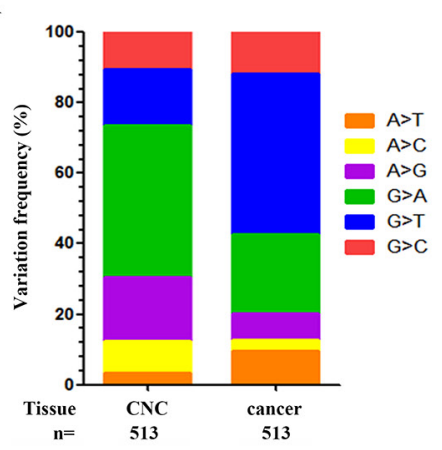

B

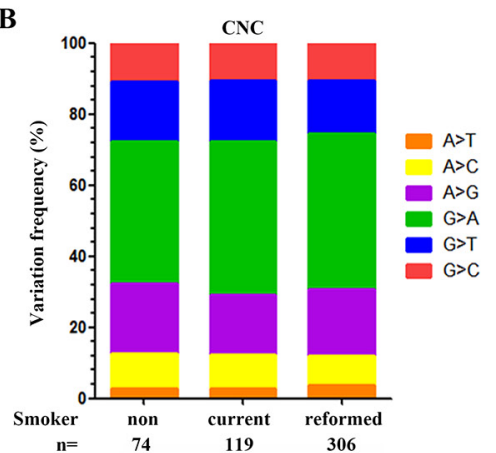

C

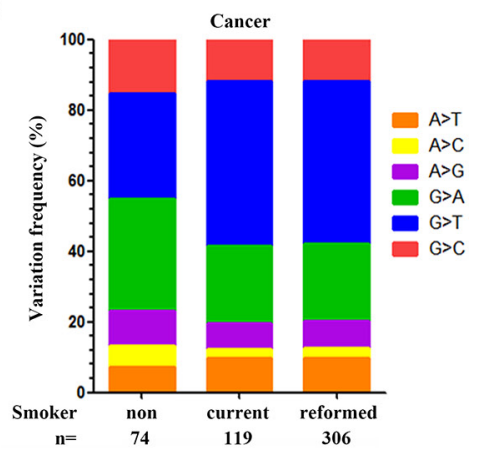

D

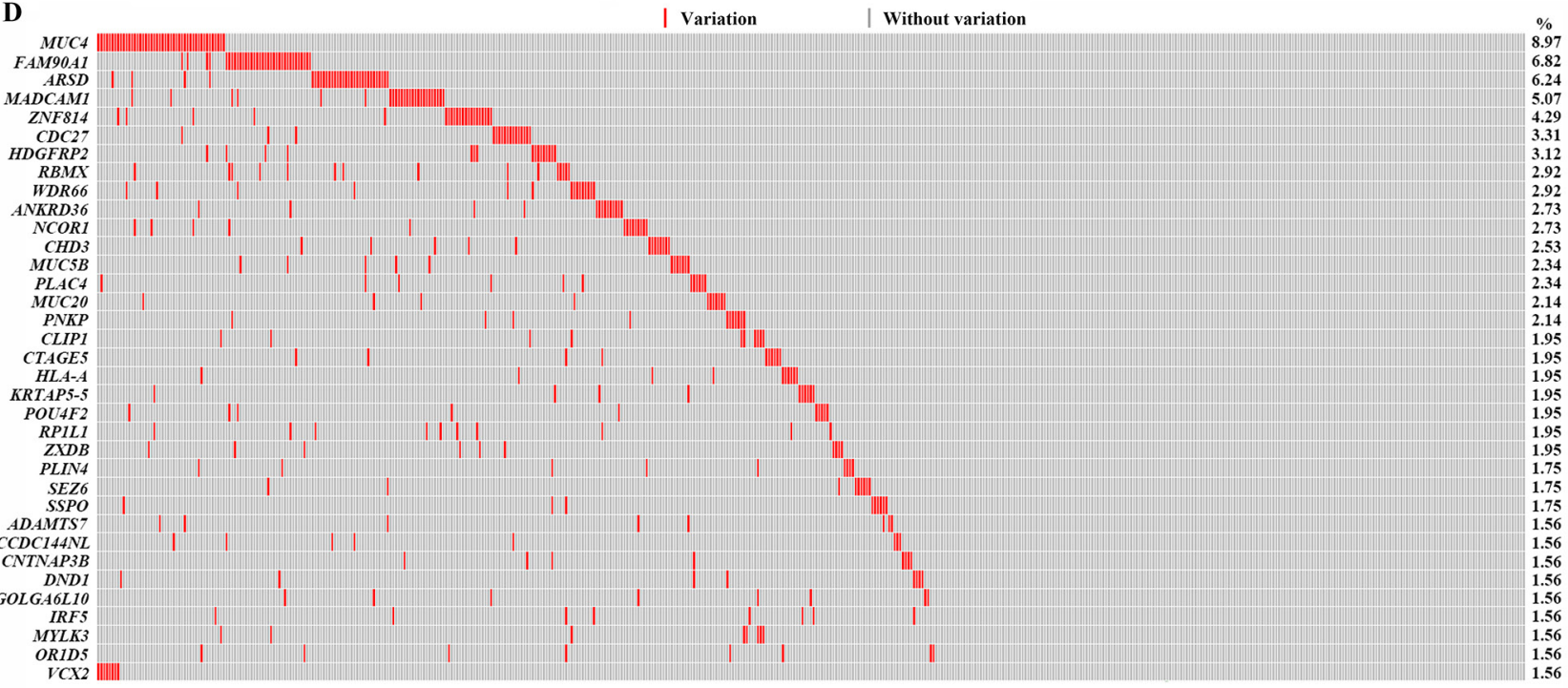

Figure 2: Genomic variations in the CNC samples. (A) The frequency of each type of single base substitution in CNCs and tumor samples of the 513 patients with LUAD. (B) The proportion of nucleotide changes in CNC samples of nonsmokers, current smokers and reformed smokers. (C) The frequency of base substitution in tumor samples of nonsmokers, current smokers and reformed smokers. (D) Significantly altered genes in CNCs of the patients. CNC samples are arranged from left to right in the top track. 
progression [17]. We found 35 types of variations distributed throughout the entire gene of $M U C 4$, including 4 recurrent ones (Figure 3). Frequent variations were found in FAM90A1 [18], in that in all 35 patients with variant FAM90A1, the nucleotide G of codon (ACG) for T344 was replaced by A and a codon for valine (GUC) was inserted in 12:8374781 position at Chr 8, leading to insertion of $\mathrm{V}$ right after T344 (Figure 3). There were 67 variations in Arylsulfatase D (ARSD) gene [19] in 32 CNCs, with S157F, G175D, and S176K substitutions found in $9(14.8 \%), 10(16.4 \%)$, and $10(16.4 \%)$ of the total variations. Twenty-nine alterations were found in MADCAM1 [20] in $26 \mathrm{CNCs}$ (Figure 3). Variations in ZNF814, CDC27, HDGFRP2, RBMX,WDR66, and $A N K R D 36$ in CNCs were also shown in Figure 3.

\section{Altered signaling pathways}

Pathway analysis was performed using the KEGG (Kyoto Encyclopedia of Genes and Genomes) database, and the results showed that genes involved in type I diabetes mellitus, allograft rejection, graft-versushost disease, autoimmune thyroid disease, antigen processing and present were the most frequently altered pathways (Supplementary Figure 3A). The GO analysis confirmed that immune response genes were altered in CNC samples, and genes associated heart, muscle, and cell development were also perturbed in CNCs (Supplementary Figure 3B).

\section{Variations associated with poor prognosis}

We analyzed the potential association between the $\mathrm{CNC}$ variations and the prognosis of the patients using the Kaplan-Meier method, and found that variations in 8 genes were associated with the prognosis of 502 patients whose survival information was available (Figure 4). Sixteen variations were found in MUC5B in 12 (2.34\%) of the 513 patients (Figure 4A). As compared with patients $(n=490)$ harboring wild type $M U C 5 B$, those with mutant transcript had much shorter overall survival ( $\mathrm{P}=0.013$; Figure $4 \mathrm{~A})$. $Z X D B$ [21] variations were seen in $10(1.95 \%)$ of the patients, whose overall survival time was shorter than those $(\mathrm{n}=492)$ with wild type $Z X D B$ (Figure 4A). Patients with variant PLIN4 [22], CCDC144NL, CNTNAP3B [23], or $C C D C 180$ [24] in CNCs also had shorter overall survival than patients with wild type transcripts (Figure 4A).

\section{Variations in CNC samples associated with favorable prognosis of the patients}

We found 14 variations in the Chromodomain Helicase DNA Binding Protein 3 (CHD3 [25]) gene in CNC samples of $13(2.53 \%)$ of the 513 patients. These
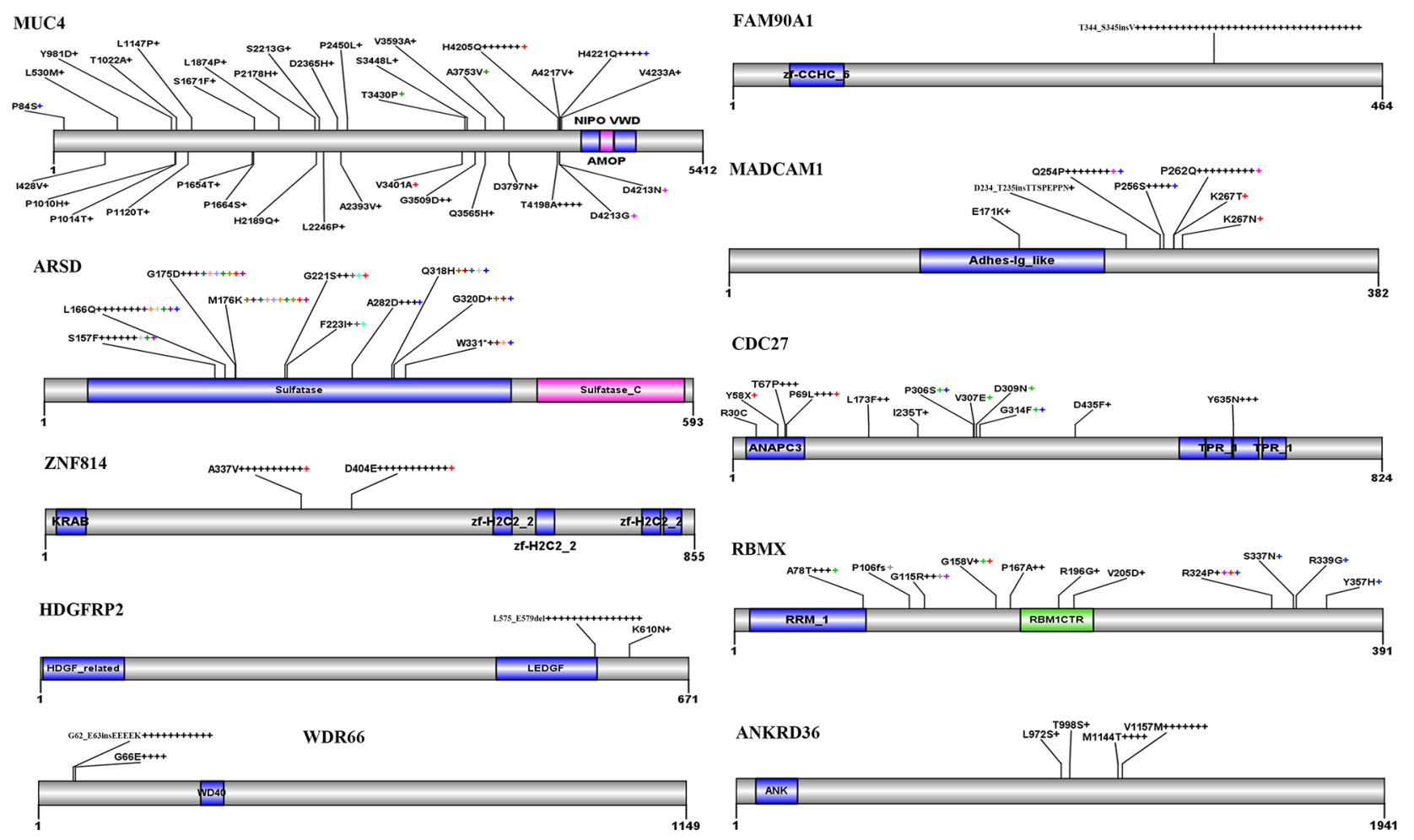

Figure 3: Recurrent variations in some representative genes in CNCs. Schematic representations of proteins encoded by the genes are shown. Numbers refer to amino acid residues. Each "+" corresponds to an independent, altered CNC sample. Variations within a gene indicated by a same-colored "+" are found in the same patient. 
variations encoded proteins with P99Lsubstitution in 1 patient, I234L substitution in 8 patients, and S237P change in 5 patients (Figure 4B). Interestingly, none of the variant $C H D 3$-bearing patients died within a followup of up to 2261 days, whereas the median overall survival of patients with wild type CHD3 (n=490) was 1492 days (range, $0-7248$ days) $(\mathrm{p}=0.047$; Figure 4B). Deletion mutations, i.e., deletion of codons for G41 G43 and G43 - A53 of the protein product encoded by KRTAP5-5 [26] gene, were found in CNCs of 10 patients. Of note, patients with variant KRTAP5-5 had longer overall survival time than those with wild type transcript $(p=0.048$; Figure 4B).

\section{DISCUSSION}

In our work characterizing somatic mutations in air pollution-related lung cancer [7], we unexpectedly noted that compared to reference human genome and cancerous tissues, the normal control lung tissues also had single nucleotide variations. This was the impetus for us to conduct this study. We used the "normal-tumor pairs" method to investigate the genomic variations in CNC samples, and reported that the CNCs did harbor genomic alterations that were confirmed by Sanger capillary sequencing of PCR products (Figure 1). These observations were validated in TCGA lung cancer
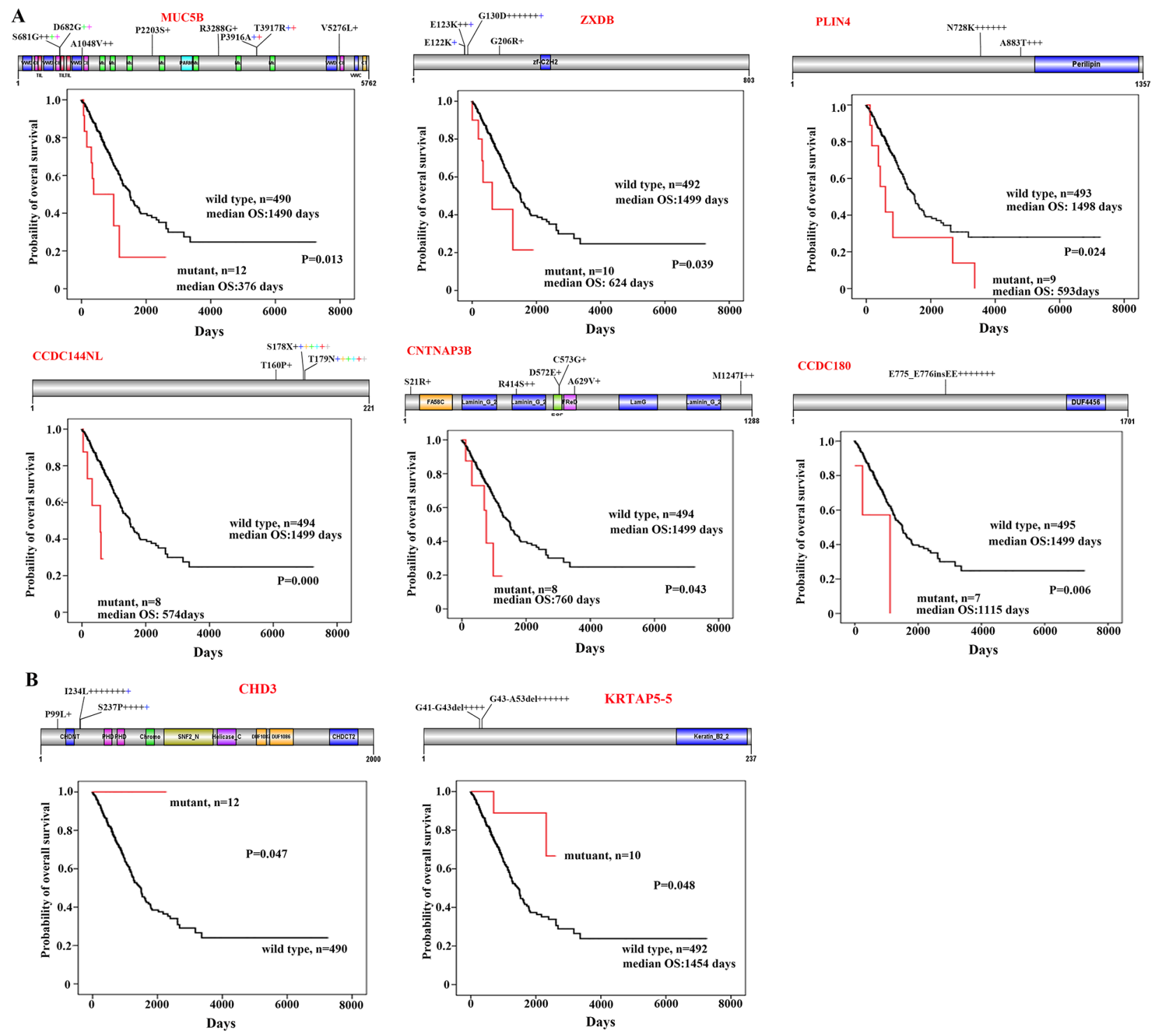

Figure 4: CNC variations associated with prognosis of the patients. (A) Variations of MUC5B, ZXDB, PLIN4, CCDC144NL, CNTNAP3B, and CCDC180 in CNCs and Kaplan-Meier curve for overall survival of the patients with wild type or variant transcripts. Schematic representations of proteins encoded by the genes are shown. (B) Overall survival of patients harboring wild type or variant CHD3 or KRTAP5-5 in their CNCs. 
genome data. In CNC genomes of TCGA datasets, the variation rates were 0.2721 exonic variations $/ \mathrm{Mb}$ and 5.2885 altered genes/sample, and the recurrent variants were of intermediate frequency ( $\sim 8.97 \%$ of the patients), whereas variations in 8 genes were associated with poor or favorable prognosis of the patients. We also dissected CNC variations in patients with lung squamous cell carcinoma, and reported a mean of 0.5661 exonic variations $/ \mathrm{Mb}$ and 7.7887 altered genes/sample in 478 patients [27]. These results uncover the previously unidentified CNC variation, and suggest a role of these alterations in lung carcinogenesis.

Some human carcinogens exhibit specific mutation signatures in the genome. For instance, PAHs which are the main carcinogens of tobacco smoke and air pollution $[5,28]$, induce the $\mathrm{C}: \mathrm{G} \rightarrow \mathrm{A}: \mathrm{T}$ substitutions in the genome $[7,15,16]$. The alkylating agent N-methyl-N-nitro-Nnitrosoguanidine (MNNG) which is found in tobacco smoke and a representative molecule of the N-nitroso compounds formed in human stomach [29], causes the $\mathrm{C}: \mathrm{G} \rightarrow \mathrm{T}: \mathrm{A}$ nucleotide changes in the genome [15]. We found that in CNCs of LUADs of both the smokers and non-smokers, the $\mathrm{C}: \mathrm{G} \rightarrow \mathrm{T}: \mathrm{A}$ transitions were the predominant nucleotide changes (Figure 2A, 2B). In tumor samples of the patients, the $\mathrm{C}: \mathrm{G} \rightarrow \mathrm{T}:$ A substitutions were the predominant nucleotide changes in non-smokers, while $\mathrm{C}: \mathrm{G} \rightarrow \mathrm{A}: \mathrm{T}$ transversions were the most frequently detected substitutions in smokers (Figure 2C). Though $\mathrm{C}: \mathrm{G} \rightarrow \mathrm{T}:$ A substitutions may be the mutational fingerprints of ageing [30], these changes in CNCs may be the result of exposure to environmental carcinogens (including second hand smoke in nonsmoker) rather than a consequence of ageing, because in this study the SNPs including those of elder individuals had been filtered out. These data further suggest the key role of environmental factors in lung carcinogenesis.

The significance of these $\mathrm{CNC}$ variations in lung carcinogenesis remains unclear. One possibility was that some of the variant genes (e.g., MUC4) were prooncogenes, cells harboring these $\mathrm{CNC}$ variations were in a "precancerous" stage, and accumulation of other mutations would result in transformation and development of malignant neoplasms. Hence, cancer may have multiclonal origins. Secondly, many of the CNC altered genes were associated with immune response (Supplementary Figure 3), which may facilitate avoiding immune destruction and cancer initiation. These possibilities warrant further investigation, and $\mathrm{CNC}$ variations in other types of cancers including cancers of oral, esophagus, stomach, colorectal, and liver, are worthy of further dissection.

Why CNC mutations were associated with prognosis represents an open question. We hypothesized that these observations may suggest the interactions between $\mathrm{CNC}$ tissues and tumor cells, constituting a micro-environment to foster or constrain cancer growth. Those $\mathrm{CNC}$ variations associated with poor outcome (MUC5B, ZXDB, PLIN4, CCDC144NL, CNTNAP3B, and CCDC180) may probably facilitate cancer initiation and progression, and may be a new clue to study cancer metastasis and evolution. $C H D 3$ encodes a chromatin-remodeling factor to repress transcription and is involved in breast cancer [31]. KRTAP5-5 is an oncogene regulating cancer cell motility and vascular invasion [32]. Whether variations in these two genes modulate transcription machinery or tumor microenvironment to constrain cancer and thus contribute to favorable prognosis, needs to be determined. In addition, how to target the $\mathrm{CNC}$ variations to develop novel therapeutics represents another open question.

\section{MATERIALS AND METHODS}

\section{Patients, whole genome sequencing, and validation by Sanger capillary sequencing}

The aim of this study was to address whether there is any genomic alteration in CNCs rather than counterpart tumor tissues (Supplementary Figure 1). This study was approved by the research ethics committee of our institute. Genomic DNAs were isolated from normal lung tissues ( $5 \mathrm{~cm}$ or more away from the tumors) and adjacent tumors of 11 patients with previously untreated LUAD, the sequencing libraries were constructed, and sequenced using the Illumina Hiseq2000 platform [7]. The genome sequences of counterpart normal lung tissues were analyzed by the Genome Analysis Toolkit (GATK) [33] UnifiedGenotyper and VarScan, compared with a reference human genome (hg19, downloaded from http://genome.ucsc.edu/) [34] and filtered against dbSNP138 common germline variants (downloaded from http://genome.ucsc.edu/) and those also detected in tumor samples. Variants from sequencing artifacts were removed by VarScan fpfilter. The following criteria were used to call variations: average base quality $\geq 20$, average mapping quality $\geq 20$, depth $\geq 6$, and variant allele frequency in counterpart sample $\geq 0.20$ and $\leq 0.05$ in tumor sample. The called variations were validated by polymerase chain reaction (PCR) and Sanger capillary sequencing using genomic DNAs of the patients and primers listed in Supplementary Table 1. Mutations in cancer genome were also analyzed by the GATK using the above criteria.

\section{Analyses of TCGA LUAD genome data}

The TCGA genome data of 513 LUADs (Table 1) were downloaded from the Cancer Genomics Hub (CGHub) (https://cghub.ucsc.edu/) with approval by the National Institutes of Health (NIH; approval number 
\#24437-4), and analyzed by GATK using the criteria for $\mathrm{CNC}$ variants.

\section{Statistics}

All of the values were evaluated using the SPSS 17.0 software for Windows (SPSS Inc., Chicago, Illinois). Statistically significant differences were determined by Student's $t$-test, and the survival curves were plotted according to the Kaplan-Meier method and compared by the log-rank test. $P$ values less than 0.05 were considered statistically significant in all cases.

\section{Author contributions}

The project was conceived and designed by GBZ. The experiments were conducted by BZ, LWQ. The genome data were analyzed by LWQ, BZ, GZW, and YC. The manuscript was written by GBZ.

\section{ACKNOWLEDGMENTS AND FUNDING}

The results shown here are in part based upon data generated by The Cancer Genome Atlas managed by the NCI and NHGRI. Information about TCGA can be found at http://cancergenome.nih.gov. The $\mathrm{dbGaP}$ accession number is phs000178.v9.p8. This work was supported by the National Natural Science Funds for Distinguished Young Scholar (81425025), the National Key Research and Development Program of China (2016YFC0905500), the National Natural Science Foundation of China (81672765), the Strategic Priority Research Program of the Chinese Academy of Sciences (XDA12010307), and grants from the State Key Laboratory of Membrane Biology. The funders had no role in study design, data collection and analysis, decision to publish, or preparation of the manuscript.

\section{CONFLICTS OF INTEREST}

No potential conflicts of interest were disclosed.

\section{REFERENCES}

1. Lawrence MS, Stojanov P, Mermel CH, Robinson JT, Garraway LA, Golub TR, Meyerson M, Gabriel SB, Lander ES, Getz G. Discovery and saturation analysis of cancer genes across 21 tumour types. Nature. 2014; 505:495-501.

2. Meyerson M, Gabriel S, Getz G. Advances in understanding cancer genomes through second-generation sequencing. Nat Rev Genet. 2010; 11:685-696.

3. Torre LA, Bray F, Siegel RL, Ferlay J, Lortet-Tieulent J, Jemal A. Global cancer statistics, 2012. CA Cancer J Clin. 2015; 65:87-108.
4. Herbst RS, Heymach JV, Lippman SM. Lung Cancer. N Engl J Med. 2008; 359:1367-1380.

5. Hecht SS. Lung carcinogenesis by tobacco smoke. Int $\mathbf{J}$ Cancer. 2012; 131:2724-2732.

6. Thun MJ, Lally CA, Calle EE, Heath CW, Flannery JT, Flanders WD. Cigarette smoking and changes in the histopathology of lung cancer. J Natl Cancer Inst. 1997; 89:1580-1586.

7. Yu XJ, Yang MJ, Zhou B, Wang GZ, Huang YC, Wu LC, Cheng X, Wen ZS, Huang JY, Zhang YD, Gao XH, Li $\mathrm{GF}, \mathrm{He} \mathrm{SW}$, et al. Characterization of somatic mutations in air pollution-related lung cancer. EBioMedicine. 2015; 2:583-590.

8. Slaughter DP, Southwick HW, Smejkal W. "Field cancerization" in oral stratified squamous epithelium. Clinical implications of multicentric origin. Cancer. 1953; 6:963-968.

9. Auerbach O, Hammond EC, Kirman D, Garfinkel L. Effects of cigarette smoking on dogs. II. Pulmonary neoplasms. Arch Environ Health. 1970; 21:754-768.

10. Gomperts BN, Spira A, Massion PP, Walser TC, Wistuba II, Minna JD, Dubinett SM. Evolving concepts in lung carcinogenesis. Semin Respir Crit Care Med. 2011; 32:32-43.

11. Kadara H, Shen L, Fujimoto J, Saintigny P, Chow CW, Lang W, Chu Z, Garcia M, Kabbout M, Fan YH, Behrens C, Liu DA, Mao L, et al. Characterizing the molecular spatial and temporal field of injury in early-stage smoker non-small cell lung cancer patients after definitive surgery by expression profiling. Cancer Prev Res. 2013; 6:8-17.

12. Ooi AT, Gower AC, Zhang KX, Vick JL, Hong L, Nagao B, Wallace WD, Elashoff DA, Walser TC, Dubinett SM, Pellegrini M, Lenburg ME, Spira A, Gomperts BN. Molecular profiling of premalignant lesions in lung squamous cell carcinomas identifies mechanisms involved in stepwise carcinogenesis. Cancer Prev Res. 2014; 7:487-495.

13. Cancer Genome Atlas Research Network. Comprehensive molecular profiling of lung adenocarcinoma. Nature. 2014; 511:543-550.

14. Imielinski M, Berger A, Hammerman P, Hernandez B, Pugh TJ, Hodis E, Cho J, Suh J, Capelletti M, Sivachenko A, Sougnez C, Auclair D, Lawrence MS, et al. Mapping the hallmarks of lung adenocarcinoma with massively parallel sequencing. Cell. 2012; 150:1107-1120.

15. Olivier M, Weninger A, Ardin M, Huskova H, Castells X, Vallee MP, McKay J, Nedelko T, Muehlbauer KR, Marusawa H, Alexander J, Hazelwood L, Byrnes G, et al. Modelling mutational landscapes of human cancers in vitro. Sci Rep. 2014; 4:4482.

16. Govindan R, Ding L, Griffith M, Subramanian J, Dees ND, Kanchi KL, Maher CA, Fulton R, Fulton L, Wallis J, Chen $\mathrm{K}$, Walker J, McDonald S, et al. Genomic landscape of 
non-small cell lung cancer in smokers and never-smokers. Cell. 2012; 150:1121-1134.

17. Karg1 A, Dinç ZA, Başok O, Üçvet A. MUC4 expression and its relation to ErbB2 expression, apoptosis, proliferation, differentiation, and tumor stage in nonsmall cell lung cancer (NSCLC). Pathol Res Pract. 2006; 202:577-583.

18. Bosch N, Cáceres M, Cardone MF, Carreras A, Ballana E, Rocchi M, Armengol L, Estivill X. Characterization and evolution of the novel gene family FAM90A in primates originated by multiple duplication and rearrangement events. Hum Mol Genet. 2007; 16:2572-2582.

19. Franco B, Meroni G, Parenti G, Levilliers J, Bernard L, Gebbia M, Cox L, Maroteaux P, Sheffield L, Rappold GA, Andria G, Petit C, Ballabio A. A cluster of sulfatase genes on Xp22.3: Mutations in chondrodysplasia punctata (CDPX) and implications for warfarin embryopathy. Cell. 1995; 81:15-25.

20. Shyjan AM, Bertagnolli M, Kenney CJ, Briskin MJ. Human mucosal addressin cell adhesion molecule-1 (MAdCAM-1) demonstrates structural and functional similarities to the alpha 4 beta 7-integrin binding domains of murine MAdCAM-1, but extreme divergence of mucin-like sequences. J Immunol. 1996; 156:2851-2857.

21. Grelg GM, Sharp CB, Carrel L, Willard HF. Duplicated zinc finger protein genes on the proximal short arm of the human X chromosome: isolation, characterization and X-inactivation studies. Hum Mol Genet. 1993; 2:1611-1618.

22. Wolins NE, Skinner JR, Schoenfish MJ, Tzekov A, Bensch KG, Bickel PE. Adipocyte protein S3-12 coats nascent lipid droplets. J Biol Chem. 2003; 278:37713-37721.

23. Boyadjiev SA, South ST, Radford CL, Patel A, Zhang G, Hur DJ, Thomas GH, Gearhart JP, Stetten G. A reciprocal translocation $46, \mathrm{XY}, \mathrm{t}(8 ; 9)(\mathrm{p} 11.2 ; \mathrm{q} 13)$ in a bladder exstrophy patient disrupts CNTNAP3 and presents evidence of a pericentromeric duplication on chromosome 9. Genomics. 2005; 85:622-629.

24. Nagase T, Kikuno R, Ishikawa K, Hirosawa M, Ohara O. Prediction of the coding sequences of unidentified human genes. XVII. The complete sequences of 100 new cDNA clones from brain which code for large proteins in vitro. DNA Res. 2000; 7:143-150.
25. Ge Q, Nilasena DS, O’Brien CA, Frank MB, Targoff IN. Molecular analysis of a major antigenic region of the 240-kD protein of Mi-2 autoantigen. J Clin Invest. 1995; 96:1730-1737.

26. Yahagi S, Shibuya K, Obayashi I, Masaki H, Kurata Y, Kudoh J, Shimizu N. Identification of two novel clusters of ultrahigh-sulfur keratin-associated protein genes on human chromosome 11. Biochem Biophys Res Commun. 2004; 318:655-664.

27. Qu LW, Zhou B, Wang GZ, Zhou GB. Genomic variations in counterpart normal controls of lung squamous cell carcinomas. Front Med. 2017. https://doi.org/10.1007/ s11684-017-0580-1.

28. Huang RJ, Zhang Y, Bozzetti C, Ho KF, Cao JJ, Han Y, Daellenbach KR, Slowik JG, Platt SM, Canonaco F, Zotter P, Wolf R, Pieber SM, et al. High secondary aerosol contribution to particulate pollution during haze events in China. Nature. 2014; 514:218-222.

29. Sugimura T, Nagao M, Okada Y. Carcinogenic action of N-methyl-N'-nitro-N-nitrosoguanidine. Nature. 1966; 210:962-963.

30. Dollé ME, Snyder WK, Dunson DB, Vijg J. Mutational fingerprints of aging. Nucleic Acids Res. 2002; 30:545-549.

31. Yu H, Jiang Y, Liu L, Shan W, Chu X, Yang Z, Yang $\mathrm{ZQ}$. Integrative genomic and transcriptomic analysis for pinpointing recurrent alterations of plant homeodomain genes and their clinical significance in breast cancer. Oncotarget. 2016; 8:13099-13115. https://doi.org/10.18632/ oncotarget.14402.

32. Berens EB, Sharif GM, Schmidt MO, Yan G, Shuptrine CW, Weiner LM, Glasgow E, Riegel AT, Wellstein A. Keratinassociated protein 5-5 controls cytoskeletal function and cancer cell vascular invasion. Oncogene. 2017; 36:593-605.

33. McKenna A, Hanna M, Banks E, Sivachenko A, Cibulskis K, Kernytsky A, Garimella K, Altshuler D, Gabriel S, Daly M, DePristo MA. The Genome Analysis Toolkit: a MapReduce framework for analyzing next-generation DNA sequencing data. Genome Res. 2010; 20:1297-1303.

34. Li H, Durbin R. Fast and accurate long-read alignment with Burrows-Wheeler transform. Bioinformatics. 2010; 26:589-595. 